\title{
Smart energy management systems: a literature review
}

\author{
N.Loganathan ${ }^{1, *}$, Dr.P.S.Mayurappriyan ${ }^{2}$, Dr.K.Lakshmi ${ }^{1}$ \\ ${ }^{1}$ Sri Krishna College of Engineering and Technology, Coimbatore, India. \\ ${ }^{2}$ Kumaraguru College of Technology, Coimbatore, India. \\ (logukirsh@gmail.com, mayurpriya@yahoo.co.in, klakshmi01@gmail.com)
}

\begin{abstract}
Recently smart energy management systems (SEMS) have been developed extremely fast. The significant methods facilitate SEMS to sustain system scheming via demand responses, possibly together with peak shaving and load changing as well as a few additional services. This paper reviews the thought of SEMS and looks into its environment. It highlights the SEMS most important apparatus and moderately analyzes a different technical approach. In adding together discuss a number of the concern and challenge as well as suggests a framework for prospect systems.
\end{abstract}

\section{INTRODUCTION}

SEMS has been in continuation in the power sector for more than a few decades. The key in function of such system are to observe, manage as well as optimize the flow and make use of power. In common, SEMS has difficult application in the production, transmission and distribution systems of the power system network. Important between the applications include demand manage and Real Time Pricing (RTP) is two ordinary techniques in Demand Side Management (DSM) projected by special energy suppliers with energy management system functionalities. SEMS is significant expansion of current for residential and industrial consumers. Demand response, demand side management, maximum shaving and demand changing which are measured to present solution to the system operative contain additional boost the constrain for more robust and intellectual SEMS. The time "smart grid" is an expression use to forename an electrical power delivers arrangement integration both digital technology and long transmission networks to optimize energy utilization and to release up innovative process for energy production as well as distribution.

\footnotetext{
*Corresponding author: logukirsh@gmail.com
} 


\section{REVIEW OF LITERATURE}

This paper gives general background of do research as well as expansion in the field of SEMS in power system arrangement based on over 20 published articles. The following release literature presents the review and relevance of each method for SEMS demand side fault in power system network. The connected assumptions completed, strengths as well as weakness of every one resolution methods are highlighted.

Ke Meng et al (2017) proposed that a challenge to organize several groups of aggregate air-conditioners for delivery system load managing. This projected method aim to present a challenge to synchronize compound group of Virtual Power Storage Space Scheme (VPSSS) to deal with complex load. A circulated manage system is future to distribute the essential dynamic control reduction among the aggregators during limited announcement to switch in order with nearby aggregators and an balance position can be met between complicated aggregators. In a distributed manage approach; the essential dynamic energy restriction can be collective amongst the participate aggregators.

Mario Collotta et al (2017) has proposed system present an Artificial Neural Network (ANN) as maintain for a Home Energy Management (HEM) arrangement base on Bluetooth low energy, called BluHEMS. The objective of infrastructure technology is to realize an extensive energy savings, in order to cut greenhouse gas emissions and to reach effectual ecological security in more than a few contexts, counting infrastructure, developed, transport, buildings, electricity generation and delivery. A smart grid is conceptualized as a grouping of underlay electrical network and superimposes communication system. In this proposed system a profound examination for the pattern of the ANN in arrange to get the one that achieve the best presentation. This system supply widespread simulative assessment, perform all the way through the Network Simulator Version-2 (NS-2), in conditions of energy utilization, demand profit, delay practiced by consumers for the planned HEM solution and in conditions of package delivery relation, delay, and jitter for the wireless networks.

Siyun Chen et al (2017) gives a human-centric Smart Home Energy Management System (SHEM) with the intention of mechanism at the "butler" plane. Based on this structure, our SHE system provide smart military to make happy the supplies of user as a butler aim not just to keep the electrical energy cost or decrease the max out load, but in addition to forecast user's stress and supervision "servants." Smart grid strategies are incorporated keen on smart home systems; it willpower be more hard and complex for user to control all strategy wisely. In this proposed system a human-centric smart home construction by the combination of considerate the behaviours of person being, infer the user's weight and utilization preference and optimally running the energy plans in smart home. The SHE system can be comprehensive for respond to a variety of require response signal and contribution expensive maintain for programming and result make at all levels of usefulness based on the people-centric framework. 
S. L. Arun et al (2017) has deals with an Intelligent Residential Energy Management System (IREMS) for producer of neat housing buildings are planned. The most important purpose of IREMS is decrease in electrical energy bills whereas maintain the power require less than the maximum demand limit subjected to the variety of constraints principal the procedure of household demand and Renewable Energy Resource (RER). Through demand manage and Real Time Pricing (RTP) is two ordinary techniques in Demand Side Management (DSM) projected by special energy suppliers. A mixed-integer linear programming based preparation algorithm whereas considers the purpose as decrease in energy consumption bill for residential energy consumers. Price profit analysis for showcasing the investments and least Cash Payback Period (CPP) predictable from the propose techniques. The resources are optimally sized with Genetic Algorithm (GA).

Daniel Minoli et al (2017) has residential the representation of energy larger than Ethernet, as measurement of an Internet of Things (IoT) -base solution, offer disrupting chance in transform the in-building connectivity of a huge swath of policy. A Building management System (BMS) is a complete platform that is working to observe and organize a building's automatic and electrical apparatus. The technical junction is as it service of IP-based end tip strategy below the power of IoT. The convergence of IoT, PoE, IP (IPv4 as well as IPv6) is predictable to improve the functionality, capability, power efficiency, and priceeffectiveness of building, affecting them up the computerization range to a "smart building" position. The expansion of cloud-based high-class analytics will facilitate international optimization and apposite data pulling out, trending, and forecasting.

Bharatkumar et al (2017) has presented to expand the Neural Network (NN) base tidy demand estimator, practical data from a real power hub managing system is use for supervise preparation. The perception of central energy management system for micro grids, base on Unit Commitment (UC) and Optimal Power Flow (OPF) model, contain been report. The optimization difficulty is solve at separate time steps taking into account reorganized forecasted input with a progressing time possibility, with obtain most constructive decision being single suitable for the next instant step. A NN based Housing Convenient Demand Profile Estimator (HCDPE) is accessible, which is urbanized by deliberate and imitation data as of a real Energy Hub Management System (EHMS).

H. S. V. S. Kumar Nunna et al (2016) has presented two power management strategies near efficiently makes use of V2G possible of Plug-in Electric Vehicles (PEVs) in supervision power imbalance in grid associated micro grids. The proposed scheme V2G combination strategies are implemented with a multi-agent arrangement developed in Java Agent progress structure and practical to a micro grid case study arrangement. The proposed cost system for fitting the PEV charge cost less than both grid connected and islanded method of process of the micro grid. The proposed approach is implementing with a Multi- Agent System (MAS) developed in Java Agent Development (JADE) structure. The projected approach is sensible to a micro grid case study scheme with two sets of information. 
Neeraj Kumar et al (2016) has presented a smart, energy-efficient system in smart grid Cyber-Physical Systems (CPSs) by means of coalition-based game theory. Mobile Cloud Networking (MCN) is a rising tools in which mobile policy are linked to a cloud server with Access Points (APs). Game is formulated connecting the smart strategy (players) and the service provider (clouds) in which together players and service providers aspire to exploit their proceeds through admiration to the accessible resources. The manage algorithms are execute in the cloud atmosphere, which is measured because the cyber plane. The proposed resolution can be implement in a real-world smart city situation for solve issue connected to demand management, frequency and voltage fluctuations at the grid.

Xin Wang et al (2016) proposed with active energy management for smart-grid powerdriven Coordinated Multi Point (CoMP) transmission. An infinite-horizon optimization difficulty is formulated to attain the most favourable downlink hand on grin formers that are robust to control reservations. Leveraging the stochastic dual-sub gradient method, expand a virtual queue base online control algorithm. Relying lying on the supposed Lyapunov optimization method as well as the exposed distinctiveness of the optimal schedules, properly launch that the proposed algorithm yields a feasible and asymptotically most favourable supply management approach for the innovative difficulty

Ganesh Kumar Venayagamoorthy et al (2016) proposed the expansion of an Intelligent Dynamic Energy Management System (I-DEMS) meant for a smart micro grid. An evolutionary adaptive dynamic programming and support learning structure is introduced for developing the I-DEMS online. To develop adaptive I-DEMS by means of an ActionDependent Heuristic Dynamic Programming (ADHDP) approach. ADHDP is base on joint concept of adaptive dynamic programming and reinforcement culture concept. The ADHDP structure employs two neural networks. I-DEMS, even as fulfilling the main goal of assembly $100 \%$ of the serious load exact requirements, still manage to advance the power dispatch to convenient loads. These resources that micro grids of the potential can be manage brightly to be self-sustainable, consistent, and ecological responsive.

Ayan Mondal et al (2015) proposed the spread Home Energy Management System with storage $(\mathrm{HoMeS})$ in a combination, which consists of compound micro grids and multiple customers, is calculated by means of the multiple-leader-multiple-follower Stackelberg game theoretic model-a multistage and multilevel game. The HoMeS model for instantaneous energy utilization of consumers in the attendance of storage space conveniences and more than a few micro grids in a combination. The first algorithm is used in the Initialization Phase (IP) for the micro grids to conclude the smallest amount of power to be generated. By the proposed advance, the distributed energy management scheme in the attendance of storage can be complete with the most favourable value of the power request by the clientele, while consider the in general energy demand in the system.

Kun Wang et al (2015) has proposed a power system through together Distributed Generators (DGs) and consumers and proposition a Distributed Location Marginal Pricing (DLMP)-based Unified Energy Management System (UEMS) representation. This 
projected system contains two parts: a game theory-based Loss Reduction Allocation (LRA); and a Load Feedback Control (LFC) with price elasticity. To developed an iterative loss decrease method by means of DLMP to compensate DGs for their contribution in energy loss reduction. This proposed system uses DLMP as a financial signal to manage and control Distribution Power Systems (DPSs). In cooperation LRA and LFC are well incorporated with a DLMP indication for the DPS.

Stefano Bracco et al (2015) proposed system to reduce the overall production expenses while fulfilling all the thermal and stimulating system constraints. To create the difficulty of supervision a micro grid entirely in conditions of an optimization problem, to present a comprehensive and inclusive model of both the mechanism and the emotional network to be insert in the optimization difficulty and mainly of all, to identify an algorithm that, in spite of its entirety, is resourceful from a computational position of observation. A competent algorithm has been resultant and obtainable to execute the optimal dispatching of low voltage micro grids. Work will believe the opportunity of remove the estimate of perfect knowledge of the weight active and immediate power and the renewable production, to acquire into description also for stochastic issues in the optimization method.

Jinsoo Han et al (2014) has proposed a smart Home Energy Management System (HEMS) structural design that considers together energy consumption and production concurrently. ZigBee base energy dimension module is used to manage the energy consumption of home appliance and illumination. A Power Line Communication (PLC) based Renewable Energy Gateway (REG) is use to check the energy age group of renewable energies. Smart HEMS constructions that consider both power consumption and invention based on ZigBee and PLC base REG correspondingly. This PLC monitor technology can supervise each planetary panel for protection. The home server cans estimation the energy production based on a conditions predict. Using the obtain energy in sequence; the home server can manage the home energy use program to reduce the power price.

Jinsoo Han et al (2014) has deals through a power line communication (PLC) obedient through Home Plug is adopt to observe each PV unit. The PLC modems are deploying on every PV component and supervise it. The Renewable Energy Gateway (REG) aggregate and provisions the monitor data starting the PLC modems and the inverter. The construction of a PV system organization base on an ordinary PLC knowledge that is fully obedient with the Home Plug Green PHY (HPGP) is projected. The PLC modems supervise every PV module. The proposed structural design is predictable to give to attractive the PV system organization and maintain the presentation of the PV system. The PLC modems and the REG, obedient with the Home Plug, can be without difficulty associated to home network base on the Home Plug.

Hung-I Hsieh et al (2014) proposed system is to create Photo Voltaic (PV) power pump constant with optimum Maximum Power Point Tracking (MPPT), which increase the PV operation and to transmit the outstanding PV energy for additional storage space in the beat break period. MPPT may be alternating to lose the track optimization. However, the 
proposed PV- Burp Charge System (BCS) not simply can formulate MPPT constant for mounting the PV utilization, but as well can transmit and store the residual PV energy for power saving throughout the pulse break time, achieve energy treasure and improvement conception. The idea of parallel charge three batteries by utilize direct renewable source create a new subject, which make it come accurate to realize the energy treasure and mending thought.

Qinran Hu et al (2013) has hardware propose of Smart Home Energy Management System (SHEMS) with the application of announcement, sense technology and mechanism learning algorithm. SHEMS is planned with sensors to identify human behaviour and after that a mechanism learning algorithm is applied to intelligently help customers decrease overall compensation on power with no or with small consumer participation. The proposed plan, customers can realize a RTP-responsive manage plan over residential loads counting EWHs, HVAC units, EVs, dishwashers, washing machines, and dryers. This paper also includes difficult and imitation consequences which show the power of the hardware coordination of the SHEMS model. If this propose can be extensively used in the potential, the manager- user arrangement will provide good potentials for electrical energy aggregators.

Zhanbo Xu et al (2013) has proposed devise the preparation difficulty of these several energy system as a Markov decision process (MDP) and at that time give two estimated resolution method. Method 1 is dynamic programming by way of price purpose estimate. Method 2 is mixed integer programming by means of value estimate. The presentation of the two methods is established on mathematical example. The consequences demonstrate that method 2 provides good solution express and with small presentation deprivation compare with method 1 . Method 2 is mixed integer programming through indicate value estimate. Numerical comparison show to facilitate method 2 provides good solution express and with little presentation poverty.

Hyung-Chul Jo et al (2013) has developed a neat heating and air conditioning forecast technique for HEMS to consider consumer handiness and individuality of thermal appliances in a smart home situation. The prototype software base on the projected technique for HEMS is as well implementing. In this proposed system describe a condensed HVAC representation that considers consumer expediency and a technique for solve the preparation problem of the HEMS with the summary HVAC reproduction. The HEMS base on the proposed technique container be used to resolve the least-cost schedule of the accessible energy resources even as minimize problem to the purchaser in a smart home surroundings.

David Chinarro et al (2013) Smart grids are a rising leaning that set up intelligence in the control grid to optimize reserve usage. This proposed system present a multi-agent arrangement representation for essential power plants, a latest power plant thought in which production no longer occur in big installation but is the consequence of the assistance of lesser and additional bright fundamentals. The ANN can nearby several diverse 
architectures, and is usually configured to study from knowledge when confront with a bottom reality recognized a priori. To facilitate be why this MAS design has to be enrich with require forecasting algorithm base on artificial neural networks to has been validate productively through actual data. Accessible forecast algorithms include be utilize to make easy prediction on a large aggregate range (nations, regions, etc.), excluding the one of propose forecasts demand at a lesser micro grid level that improved hysterics the VPP range.

\section{CONCLUSION}

In this paper, an overview and key issues of different research studies for the instant prospect of implement an energy management system is not through entity customers. Though, by the aggregation as well as incorporation of some consumers present is an elevated prospective. Utilities and SEMS manufacturers require to line to put together components and make certain level utility household contact. Efficient top-down incorporation is suggested. This resources shift from centralized systems to grid or power plants to aggregation of buildings as well as to end with to individual buildings. SEMS is additional than transfer of information and further more than switching on and off strategy. Generally, goals for the completion of SEMS should be obviously definite and sub-defined for the range of concentration groups such as researchers, government, manufacturers and consumers.

\section{References}

1. Ke Meng, Zhao Yang Dong, Zhao Xu, IEEE Transactions On Systems, Man, And Cybernetics: Systems, pp.1-10, (2017).

2. Mario Collotta, Giovanni Pau, IEEE Transactions On Green Communications And Networking, Vol. 1, no. 1, pp. 112-120, (2017).

3. Siyun Chen, Ting Liu, Feng Gao, Jianting Ji, Zhanbo Xu, Buyue Qian, Hongyu Wu, and Xiaohong Guan, IEEE Communications Magazine pp. 27-33, (2017).

4. S. L. Arun, M. P. SelvanIEEE Systems Journal pp.1-12, (2017).

5. Daniel Minoli, Kazem Sohraby, Benedict Occhiogrosso, IEEE Internet Of Things Journal Vol. 4, no. 1, pp.269-283, (2017).

6. Bharatkumar V. Solanki, Akash Raghurajan, Kankar Bhattacharya, IEEE Transactions on Smart Grid Vol. 8, no. 4, pp.1739-1748, (2017).

7. H. S. V. S. Kumar Nunna, Swathi Battula, Suryanarayana Doolla, IEEE Transactions on Smart Grid, DOI 10.1109/TSG.2016.2646779, pp.1-12, (2016).

8. Neeraj Kumar, Sherali Zeadally, and Subhas C Misra, IEEE Wireless Communications, pp.100-108, (2016).

9. Xin Wang, Yu Zhang, Tianyi Chen and Georgios B. Giannakis (2016), IEEE Journal on Selected Areas in Communications, Vol. 34, no. 5, pp.1348-1359.

10. Ganesh Kumar Venayagamoorthy, Ratnesh K. Sharma, Prajwal K. Gautam and Afshin Ahmadi, IEEE Transactions on Neural Networks and Learning Systems, Vol 27, no. 8, pp.1643-1656, (2016).

11. Ayan Mondal, Sudip Misra and Mohammad S. Obaidat, IEEE Systems Journal, pp.110, (2015). 
12. Kun Wang, Zhiyou Ouyang, Rahul Krishnan, Lei Shu and Lei He, IEEE Transactions on Industrial Informatics, Vol. 11, no. 6, pp.1607-1616, (2015).

13. Stefano Bracco, Massimo Brignone, Federico Delfino and Renato Procopio, IEEE Systems Journal, Digital Object Identifier 10.1109/JSYST.2015.2419273, pp.1-11 (2015).

14. Jinsoo Han, Chang-Sic Choi, Wan-Ki Park, Ilwoo Lee and Sang-Ha Kim, IEEE Transactions on Consumer Electronics, Vol. 60, no. 2, pp.198-202, (2014).

15. Jinsoo Han, Chang-Sic Choi, Wan-Ki Park, Ilwoo Lee, and Sang-Ha Kim, IEEE Transactions on Consumer Electronics, Vol. 60, no. 2, pp.184-189, (2014).

16. Hung-I Hsieh, Cheng-Yuan Tsai, and Guan-Chyun Hsieh, IEEE Transactions on Power Electronics, Vol. 29, no. 4, pp.1777-1790, (2014).

17. Qinran Hu and Fangxing Li, IEEE Transactions on Smart Grid, Vol. 4, no. 4, pp 1878-1887, (2013).

18. Zhanbo Xu, Qing-Shan Jia, Xiaohong Guan, Jianxiang Shen, IEEE Transactions on Automation Science and Engineering, Vol. 10, no. 3, pp 603-614, (2013).

19. Hyung-Chul Jo, Sangwon Kim, and Sung-Kwan Joo, IEEE Transactions on Consumer Electronics, Vol. 59, no. 2, pp 316-322.

20. Jaime Lloret, David Chinarro, Jorge J. Gomez-Sanz, IEEE Communications Magazine, pp 106-113. 